die Verschlüsselung von Telegram sei nicht so sicher, wie angenommen. Offen gesagt weiß ich es nicht. Ich habe technische Argumente in beide Richtungen gehört. Tatsache ist, dass es das am besten vor Eingriffen durch Regierungen oder Unternehmen geschützte System ist, und zu einem der meistverwendeten Messengerdienste zählt. Zurzeit zählt es 200 Millionen Nutzer weltweit, die jährlich um 50 \% ansteigen. (Castells 2018)

Angesichts der Zielstrebigkeit der russischen Regierung und neoliberaler Regime zur Kontrolle des politischen Aktivismus und der damit verbundenen Naturalisierung des hegemonialen Systems entwickeln zivile Gegenbewegungen Ressourcen, die diese Regierungen gewaltig ins Stolpern bringen. Dabei ist es nicht ihre Absicht, die zentralisierte Kontrolle insgesamt zu beenden. Ein Grund dafür ist die Verknüpfung ihrer Einrichtungen mit so genannten sektoralen Staatsbürgerschaften - etwa in den Bereichen Frauen, Jugend, oder Migration. Angesichts der Einsicht, dass es unmöglich ist, das gesamte Überwachungssystem abzuwickeln, beschränken sie sich darauf, Netzwerke jenseits der zentralen politischen Macht, der durch kommerzielle Interessen und Werbung dominierten Digitalwirtschaft, sowie des Datenhandels aufzubauen. Castells zweifelt die Wirksamkeit der alternativen Verschlüsselung an. Ebenso stellt sich die Frage, ob die alternativen Abspaltungen von der dominanten Kommunikationsökonomie nicht implizit zur deren Naturalisierung beitragen, die wir dann nicht mehr rückgängig machen können.

\title{
Protestbewegungen - neue Parteien oder unabhängige Konfrontation?
}

Die sozio-technologischen Innovationen wirken auf mehreren Ebenen. Sie bieten Möglichkeiten zur Erneuerung der Kommunikation innerhalb einer Region oder einem Land und besetzen öffentliche oder private Räume und Netzwerke. Sie verbinden Nutzer mit internationalen Kreisläufen. Es entstehen Möglichkeiten, um Selbstermächtigung durch Solidarität aus den Erfahrungen anderer zu lernen. Darüber hinaus können die Reichweite der Botschaften und der soziodigitalen Strömungen zu einem Teil unserer Körper und unserer sozialen Beziehungen zueinander werden. Wir können eine neue Freizügigkeit erreichen, ohne dass Parteien oder institutionelle Trägheit diese einschränken. Wir haben gesehen, dass eine Erschütterung des patriarchalischen Systems selbst unter konservativen Rechtssystemen möglich ist, 
indem wir für die Entkriminalisierung von Abtreibungen, gegen sexuelle Belästigung oder Frauenmorde mobilisieren. Aktuelle Beispiele sind Argentinien, Brasilien, Chile, Mexiko, und andere. Kreativschöpfer finden Wege, um ihre Arbeit außerhalb der Bürokratisierung von Museen oder über die Unternehmensstrukturen des Fernsehens oder der Internetdienstleister hinweg zu kommunizieren. Soziale Bewegungen schaffen kooperative Verbindungen, die ihre relative Autonomie dank offen zugänglicher Technologien verbessern. Dies stellt einen neuen Weg zum Gemeinsamen dar. Rossana Reguillo bezeichnet diese Phänomene als »erweiterten öffentlichen Raum« und als »Zonen der affektiven Intensivierung «. Es handelt sich um nicht konditionierte Konversationen, die zwar häufig ausspioniert und über längere Zeit gespeichert werden, die aber trotzdem ein Schutzschild über Handlungen des zivilen Ungehorsams gegen fremdbestimmte Programmierungen spannen.

Auf welche Weise erfinden diese soziokulturellen Mutationen die Staatsbürgerschaft neu? Sind sie mehr als eine überraschende Wendung in der Krise des Establishments? Beschränken sie sich nicht auf kurzlebige Mobilisierungen, beziehungsweise auf Arten der gegenseitigen Begleitung durch Anstrengungen und Niederlagen, in denen man sich gegenseitig tröstet? Verändern sich nur die Vorstellungen, den Horizont des Möglichen und einige Bräuche?

Wir wissen bereits, dass sich nach dem Verschwinden von 43 Studierenden am 26. September 2014 in Iguala Hunderte von jungen Menschen zusammen mit den Eltern der Opfer mobilisierten. Dies geschah im Anschluss an »YoSoy132«, das zu dem Zeitpunkt längst erloschen war. In der Bewegung machten sich die Jugendlichen mit der Organisation von Protestmärschen vertraut und entdeckten das Potenzial der Netzwerke gegenüber dem Fernsehen zur Untersuchung von Vertuschungsversuchen oder Beschönigungen der Regierung.

Natürlich spielt auch die Tatsache des Regierungswechsels ab Dezember 2018 eine Rolle. Unter dem Präsidenten Andrés Manuel López Obrador wurden die Archive wieder geöffnet und die Eltern der Verschwundenen wurden im Nationalpalast empfangen. Eine offizielle Kommission wurde zur Öffnung der Gräber entsandt. Die Aufdeckung der wahren Geschehnisse wurde wieder zur Aufgabe öffentlicher Institutionen. Zu Beginn des Jahres 2019 beschäftigten sich hochrangige mexikanische Regierungsbeamte mit vernachlässigten Fällen oder Vertuschungen. Mitte des Jahres gaben sie allerdings dem Druck der USA nach und wiesen zentralamerikanische Migranten aus. Die Grenze zu Guatemala wurde remilitarisiert und folglich mehr Budget für die Zurückhaltung von Ausländern bereitgestellt, als für die Aufarbeitung der Op- 
ferrechte. Die Regierung zog auch die staatliche wirtschaftliche Hilfe zurück, die zur Unterstützung zivilgesellschaftlicher Organisationen und für deren Beratungsarbeit für die Angehörigen der Vermissten vorgesehen war.

Dies führt uns zur Rolle derjenigen Bewegungen, die die zivilen Anstrengungen der letzten vier oder fünf Jahrzehnte trotz der Veränderungen in der Regierungspolitik und der offenen Feindseligkeit der Behörden und anderer Zivilsektoren unterstützt haben: Bewegungen, die auf einer Aufklärung der in der Diktatur geschehenen Genozide beharren. Sie möchten das Verschwindenlassen militanter und gewöhnlicher Bürger durch kriminelle Organisationen und Polizei- und Militäreinheiten unbedingt aufarbeiten. Es gibt keine radikalere Form der Entbürgerlichung als die Ermordung derer, die sich auf ihre Rechte berufen - seien es ökologische, soziale oder territoriale. Sie gipfelt in der anschließenden fehlenden Dokumentation ihres Todes und im aktiven Verhindern der Aufarbeitung der Verbrechen.

In diesem Zusammenhang sticht die Arbeit des Equipo de Antropología Forense hervor. Es handelt sich um einen Zusammenschluss aus NGOs, der vor 35 Jahren in Argentinien seine Arbeit begann, seitdem in Chile, Guatemala, Mexiko und Uruguay weiterentwickelt wurde und auch in afrikanischen, asiatischen und europäischen Ländern tätig war. In ihren Studien gelang es der Organisation, 780 Vermisste in Argentinien zu identifizieren und schwerwiegende Staatsverbrechen aufzuklären, darunter das an den Studierenden im mexikanischen Ayotzinapa. Mit ihrer Arbeit gelingt es ihnen, die Praxis der Straflosigkeit zu durchbrechen. So lautet auch der Titel eines Buches unter der Herausgeberschaft von Silvia Dutrénit (2017). Ihre Veröffentlichung systematisiert die Erfahrungen in den genannten Ländern und die Selbstwirksamkeit der Bürger angesichts der Verzerrungen und Vertuschungen durch den Staat. Indem sie von Schweigen und Verwirrung geprägte Orte erhellen gelingt es der Organisation, dass Mütter, Großmütter und andere Verwandte wieder lernen, Vertrauen zu fassen. Das gilt ebenso für zahlreiche Bürger, die auf die Straße gehen, um weiter ihrer Arbeit nachgehen zu können und dies im Sinne der sozialen Absicherung bei der Regierung einfordern.

Im Folgenden eine Transkription von Interviewauszügen aus dem Mai 2019 mit Luis Fondebrider, Präsident des Equipo de Antropología Forense:

Haben Sie sich mit der Zeit daran gewöhnt, so viele Leichen zu sehen?

Die Arbeit mit den Familienmitgliedern ist viel schwieriger, als die Ausgrabung der Leichen. Es fällt nicht leicht, sich die Erzählungen der Suche von den Eltern anzuhören. An vielen Orten haben wir Menschen gehört, die acht 
oder zehn Familienmitglieder vermissen und uns erzählen, wie sie während ihrer Suche die persönlichen Gegenstände oder das Bett dieser Familienmitglieder so lassen, wie sie waren. Das ist heftiger als das Ausheben der Leichen. Den Angehörigen zuzuhören ist die heftigste Sache, die der Arbeit aber gleichzeitig Bedeutung verleiht.

Können die Angehörigen durch das Finden der Leiche ein Kapitel schließen und den Schmerz loslassen?

Ich glaube nicht, dass die Angehörigen jemals mit der Angelegenheit abschließen werden, aber es mildert ein wenig den Schmerz und die emotionale Qual. Die Tatsache, dass ihr Verwandter gefunden wird und eine Ruhestätte findet, etwa mit Blumen oder einer Cedenktafel, stellt sicherlich einen Wandel dar. Trotzdem kehrt ihr Familienmitglied nie wieder zurück. Die Familien sagen uns, dass die Beerdigung ihnen guttut, und das ist für uns die größtmögliche Zufriedenstellung. (Ginzberg 2019)

Das Equipo de Antropología Forense legt großen Wert auf die wissenschaftliche und die transdisziplinäre Arbeit. Über eine Verknüpfung mit heterogenen Daten wird versucht, die jeweilige Bedeutung jeder verschwundenen Person zu priorisieren:

Das Neueste ist ein Arbeitsbereich der neuen Technologien, in dem wir Anwendungen und Methoden nicht-traditioneller wissenschaftlicher Disziplinen im forensischen Bereich nutzen, wie zum Beispiel die Physik und die Mathematik. Wir führen anspruchsvollere Analysen durch, die zum Beispiel die Mengenlehre implizieren und nach Prioritäten funktionieren: etwa wie eine Person an einem Ort verschwindet, den wir dann mit anderen Verschwundenen in Verbindung bringen. Damit wagen wir uns auf neues Terrain, das nicht direkt etwas mit dem Suchen nach Verschwundenen auf einer Liste oder an einem Ort zu tun hat, sondern eine etwas abstraktere Analyse beinhaltet [...] Wir haben es mit geographischen Räumen, Daten und Personen ohne politische Zugehörigkeit, und mit Personen mit politischer Zugehörigkeit zu tun. Mit der Hilfe eines Physikers setzen wir diese Personen durch numerische Parameter miteinander in Beziehung. So entsteht ein Zusammenhang zwischen der Bestimmung von Prioritäten und Werten und der jeweiligen Repräsentation jeder Person in diesem Beziehungsnetz. Wir hoffen, dass diese neue Arbeitsweise Früchte tragen wird. Dabei soll das Potenzial der Wissenschaft ausgeschöpft werden. In Argentinien gibt es eine große wissenschaftliche Kapazität, und es gibt Menschen, die das Problem 
aus einem anderen Blickwinkel denken können. Die Fälle, mit denen wir uns beschäftigen, sind komplex und massiv. Die Leichen werden vorsätzlich versteckt, und es werden keine Informationen rausgegeben. Deshalb brauchen wir andere Instrumente als die traditionellen, um weiterzukommen. Wir müssen das Problem von einer anderen Seite denken (Idem).

Die Testimonios und Analysen zu den Menschenrechtsbewegungen zwingen uns zur Überprüfung gewöhnlicher Denkkriterien zu Bürgeraktionen durch einen intersektionalen Ansatz zwischen kurzlebigen Bewegungen, unflexiblen Parteien, Regierungen, Unternehmen, kriminellen Vereinigungen, Medien und sozialen Netzwerken. Die technologischen Ressourcen wiederholen die klassische Diskussion zu der Frage, ob sich zivile Bewegungen zwecks einer größeren Effektivität als neue Parteien innerhalb institutioneller Strukturen organisieren müssen - das heißt Präsidentschaft, Landesregierungen, Parlamente, Bürgermeisterämter. In diesen Ämtern wird die politische Steuerung mittel- und langfristig entschieden. Gesetze oder die für sie zuständigen Organe werden reformiert. Die durch Chats, Netz-Solidarität und Hacktivismus bewirkten Aktionen erreichen zwar ihren Störeffekt, aber sie kommen auch an ihre Grenzen: Erstens eignen sich etablierte politische Kräfte diese gleichermaßen an. Zweitens ist die von ihnen hervorgebrachte virtuelle und reale Veränderbarkeit der Sozialisation durch die Prekarität der Arbeitsbedingungen, der Wirtschaftslage und der projektabhängigen Lebensrealitäten bedingt.

Diese Spannung zwischen Bewegungen und Parteien hatte ein anderes Format in Zeiten, als gewerkschaftliche Forderungen noch an den Rahmen der Kontinuität von Unternehmen und Arbeitsrechten angelehnt waren, die die Arbeitnehmerrechte mehr oder weniger für mehrere Jahre sicherten. Diejenigen Bewegungen, die aus ehemaligen Gewerkschaften hervorgegangen sind - darunter die brasilianische PT - wurden zu Parteien oder kämpften gegen korrupte Establishment-Parteien im Sinne einer Rückgewinnung des Sozialen. Ähnliches gilt für indigene Bewegungen während der Epoche des Fortschritts zur Anerkennung ihrer Rechte durch die Nationalstaaten, die heute weniger Macht und weniger Möglichkeiten als je zuvor haben, ihre Rechte geltend zu machen. Viele Gewerkschaften und Parteien wurden geschwächt oder existieren bloß noch in Form einer Karikatur. Sie sind abhängig von transnationalen Dynamiken, denen sie sich nicht entgegenstellen können oder wollen. Was wir beinahe schon routinemäßig Krise der Demokratie nennen ist größtenteils ein komplexer und multidimensionaler Machttransfer hin zu In- 
stanzen, die weit entfernt von den Bürgern agieren. Die Gesetze, die Stimmrechte festlegten und die Beteiligung derer schützten, die sich in ihrer Rolle als Arbeiter, Angestellte, Frauen oder Indigene zivilpolitisch einbrachten, wurde von einer neuen wirtschaftlichen und soziokulturellen Ordnung überholt.

Die Parteien behalten jedoch die Zustimmungsquoten, wirtschaftliche und kommunikationsbezogene Ressourcen und die Fähigkeit, Allianzen zu bilden. Das sind nur einige der Gründe, weshalb manche Bewegungen den Weg zur Partei gehen. Wie wir in der Entwicklung von "YoSoy132« beobachten konnten, war deren Verweigerung gegenüber den Anstößen von Politikern und liberalen Politologen zur Organisationsform als Partei klug. Man hatte der Bewegung dazu geraten, ihre Forderungen zum Teil des Parteiensystems zu machen, ohne dabei die eigentliche Unzufriedenheit und das aufständische Potenzial des Phänomens zu erkennen. In einem anderen Kontext als dem in Mexiko, nämlich in Spanien, wählte die Bewegung "M15 « den für sich passenden Moment aus, um ihre Umwandlung in Podemos zu verkünden und Gesetzgeber und Bürgermeister auf ihre Seite zu ziehen. Der ursprüngliche Aufstand wurde im Rahmen eines ausgefeilten Programms gestärkt und Veränderungen für die Gesellschaft als Ganzes erreicht. Rechte, die die Konservativen abzuschaffen planten, wurden bewahrt. Diese bezogen sich etwa auf digitale Praktiken. Schließlich konnte eine gesetzliche Anerkennung erreicht werden und Entscheidungen der digitalen Regierung (egobierno) wurden berücksichtigt.

In Mexiko setzten einige junge Teilnehmer aus der »YoSoy132«-Bewegung auf die Ernennung unabhängiger Kandidaten in mehreren Gebieten des Landes. Wikipolítica unter der Führung von Pedro Kumamoto erzielte die besten Wahlergebnisse. Er hatte es mit einer Kampagne unter sehr geringen finanziellen Mitteln zum neuen Senator in Jalisco gebracht. Bei den nationalen Wahlen 2018 wagte man dann ein größeres Unterfangen: Die von ihm geleitete Plattform präsentierte neun unabhängige Kandidaten für den lokalen Kongress, und fünf für die föderale Ebene, unter einem gemeinsamen Programm. Das Ergebnis von weniger als einer Million Stimmen wurde anschließend auf das Fehlen ausreichender finanzieller Mittel zurückgeführt, die einen echten Wettbewerb mit der Wahlkampfmaschinerie und den Tricks des traditionellen Parteiensystems unmöglich gemacht hatte. Kumamoto schrieb dazu, dass "uns dadurch bewusstwurde, wie vergänglich unabhängige Kandidaturen im Gegensatz zu unseren Absichten sind: Wir wollen eine Institution gründen, 
die über die Wahlen und den Personenkult hinaus geht« (Kumamoto 2019, 33).

Die Bewegungen der letzten Jahrzehnte oszillierten zwischen diversen Formaten, wie zum Beispiel die vom Mai 1968, bei der französische Studierende sich mit Arbeiter-Gewerkschaften und Parteien verbündeten (und auch auf unüberwindbare Meinungsverschiedenheiten stießen). Im selben Jahr gab es in Mexiko, und ein Jahr später in Argentinien demokratisierende Allianzen. Seitdem mehren sich in Lateinamerika die Beispiele für sektorübergreifende und verflochtene gemeinsame Initiativen: Mexikanische Studierende arbeiten mit indigenen Zapatisten zusammen, argentinische Feministinnen vereinen sich mit Arbeitslosen und aufständischen Fabrikarbeitern, und mit Familien von Vermissten. Was sie gemeinsam haben, ist die soziale Schieflage, die durch eine Wirtschaftspolitik verursacht wurde, die das Einkommen der großen Mehrheit abwertet. Diese Bündnisse sind oft kurzlebig oder sie betreffen nur bestimmte Bereiche. So schafften einige Bewegungen in Brasilien den Sprung in die Mittelschicht (während der Regierungszeit von Lula circa 40 Millionen), während andere empörten oder frustrierten Randgruppen die PT ablehnten und ihre Stimme Bolsonaro gaben.

Der Hegemoniebegriff und die Artikulation soziokultureller Unterschiede werden in mehreren Ländern Lateinamerikas und mit unterschiedlichen Ergebnissen neu definiert: Hierzu verweise ich auf das Buch von Alejandro Grimson (2019), in dem der seit vielen Jahren anhaltende Peronismus analysiert wird. Seine Untersuchung sieht diesen als Ergebnis eines Zusammenspiels vieler sehr verschiedener Zielvorschläge unter der Verknüpfung verschiedener kollektiver Subjektivitäten. Die hegemoniale Kapazität im Sinne einer Artikulation von Allianzen zwischen Gewerkschaften, Arbeitgebern, regionalen Führungsfiguren, Intellektuellen, urbanen und popularen Bewegungen mit unterschiedlichen Zielen ist eine maßgebliche Kraft bei der gesellschaftlichen Neugestaltung. Zum Beispiel haben solche Bewegungen es geschafft, der Opposition ihren sozialen Sinn wieder zu verleihen. Gleichzeitig ist sie auch ausschlaggebend für ihre Instabilität. Unter den vielen Strategien zur erfolgreichen Analyse in Grimsons Buch stößt der Leser auch auf einen ebenso elementaren, wie vernachlässigten Aspekt: »Der Peronismus wäre mit seinen Charakteristika nicht ohne die spezifischen Merkmale des Anti-Peronismus entstanden « (Grimson 2019, 13). Genauso wie es verschiedene Formen des Peronismus gibt - die sich mit der Zeit verändern - gibt es auch verschiedene Anti-Peronismen. Analog dazu ergeben sich die kommunikationsbezogenen Hegemonien, die diese (Anti-)Peronismen in jeder histori- 
schen Periode die Möglichkeit zur Machtteilhabe verleihen, aus der Fähigkeit zur Verbindung mit heterogenen Sektoren. Wir können davon ausgehen, dass ihr relativer Erfolg bei der Aufrechterhaltung und Steigerung ihres hegemonialen Potenzials davon abhängig ist, wie sie sich wirtschaftlich und technologisch umstellen oder anpassen, ob sie mit Unternehmen zusammenarbeiten, oder ob sie neue Gewohnheiten und Neigungen der Öffentlichkeit, beziehungsweise der Nutzer wahrnehmen und aufgreifen. Zu der ursprünglichen Heterogenität hinzu kommt die Anpassung an heterogene Zeitverhältnisse.

Wir beobachten, dass Akteure sich bei ihrer Verbindung mit Kommunikationsmedien oder Netzwerken in einer chamäleonartigen Kunst üben, ganz ähnlich zu den Parteien, die ihre Ästhetik an die der unabhängigen Bewegungen anpassen. Der Bürger-Verbraucher-Nutzer regt diese Mutationen manchmal selbst an und tut sich in ihrer Erkennung schwer. Im anderen Fall tut er sich mit den anderen Bürgern zusammen und erlernt sozusagen die Entlarvung dessen, was nur eine Farce, was eine Möglichkeit, und was eine Bedrohung darstellt.

Wie zuvor erwähnt sind die Korruption, die zunehmende Bürokratisierung und die mediale Distanzierung der Parteien gleichzeitig ein Grund für deren Niedergang und ihre Blindheit zur Wahrnehmung neuer kommunikativer Heterogenitäten. In Bezug auf die Bürger begrenzen sich die Medien und die Parteien auf ihre Fähigkeit zur Vermittlung, indem sie die legitime Heterogenität der Widerstände abwerten. In den Transformationsbewegungen selbst wurde die Schwierigkeit der Konsensfindung angesichts der Heterogenität schon seit Anbeginn viel debattiert: Es wurde gesagt, der Feminismus solle mehr sein, als ein Ausdruck des Womanismus nach Marta Lamas; Ethnizismen werden als Verteidigung des Eigenen gerechtfertigt, aber begrenzt, sobald sie sich in Fremdenfeindlichkeit verwandeln oder die Verflechtung wirtschaftlicher, kultureller und geschlechterbezogener Unterdrückung außer Acht lassen.

Die ungelösten Schwankungen zwischen Bewegungen und Parteien entstehen durch Mängel in Bündnissen, durch die man in manchen Fällen die Heterogenität anerkennt, ihr dann aber schnell wieder eine Absage erteilt. Die gegenwärtige Politik weist zahlreiche große Schwierigkeiten bei der Aufrechterhaltung von Vereinbarungen auf, bei denen Utopien durch Differenzen, sowie durchaus durch die Sabotage selbstverliebter Partei- und Gewerkschaftsführer geprägt werden, welche die Medien und Netzwerke unterdrücken und kooptieren, sobald sie ihnen widersprechen. 